\title{
La communication financière des collectivités territoriales : nouveaux usages de l'Internet pour un système de financement en mutation ${ }^{1}$ \\ Muriel Michel-Clupot ${ }^{a^{*}}$ et Serge Rouot ${ }^{b}$ \\ a Maître de Conférences en Sciences de Gestion \\ Cerefige - IUP Finance - Université de Lorraine \\ 13, place Carnot - CO 70026 - 54035 Nancy Cedex \\ ${ }^{b}$ Maître de Conférences en Sciences de Gestion \\ Cerefige - Isam-IAE - Université de Lorraine \\ 25, rue Baron Louis - CS 10399 - 54007 Nancy Cedex
}

\section{Résumé}

Un recours accru au financement par les marchés suppose pour les collectivités territoriales une communication financière sur Internet. Une évaluation des pratiques actuelles des collectivités émettrices et non émettrices selon une double méthode - scoring et analyse textuelle - révèle peu de différences significatives dans le contenu et l'utilisation du Web. Une plus grande interactivité est préconisée. Un clivage «communication budgétaire/financière » émerge pour expliquer les divergences dans l'exploitation des potentialités d'Internet.

(c) 2013 IDMP/Lavoisier SAS. Tous droits réservés

Mots clés : communication financière, Internet, collectivités territoriales, scoring, analyse textuelle.

\section{Abstract}

Local governments' financial communication: new uses of the Internet for a changing finance system. For local governments, increasing capital market financing implies financial communication on the Internet. The assessment of the current practices (i.e. scoring and textual analysis) of bond issuers and non-issuers reveals few significant

\footnotetext{
${ }^{1}$ Cet article a fait l'objet d'une communication lors du colloque du Groupement de recherche sur I'administration locale en Europe (Grale-CNRS), intitulé « Nouveaux usages de I'Internet dans les collectivités territoriales ». Organisé par Jacques Spindler et David Huron, ce colloque s'est tenu à I'IAE de I'Université Nice-Sophia Antipolis les 24 et 25 novembre 2011.
}

*Auteur correspondant : muriel.michel@univ-lorraine.fr doi:10.3166/pmp.30.159-180 @ 2013 IDMP/Lavoisier SAS. Tous droits réservés 
differences in the content and the use of the Web. Greater interactivity is recommended. A split between budgetary and financial communication tends to explain the differences in exploiting the Web's potential.

(c) 2013 IDMP/Lavoisier SAS. Tous droits réservés

Keywords: Financial communication, Web, local governments, scoring, textual analysis.

«Parce qu'il porte notre parole et notre écriture, Internet façonne notre rapport au sens, il est [...] le miroir numérique dont le reflet donne à voir notre manière d'être » N. Curien, W. Maxwell, 2011.

\section{Introduction}

Les crises financières à répétition bousculent le système de financement des collectivités territoriales françaises. La relation banques-collectivités est en profonde mutation : 2008 révèle la toxicité de certains produits structurés, 2011 assiste au démantèlement de leur premier prêteur. Dans ce contexte troublé, les transferts de compétences renforcent encore les besoins de financement de l'échelon local. Les élus officialisent alors la volonté collective de création d'une agence de financement des investissements locaux et un recours accru au marché se dessine. Une communication financière des collectivités locales, sur le modèle de celle des sociétés, doit alors être mise en place, notamment au travers de sites Internet performants.

Les lois de décentralisation ont créé plusieurs injonctions communicationnelles, de la construction d'un « sentiment identitaire » (Megard, Deljarrie, 2009 : 21) à une communication de proximité et participative. Reposant sur des pratiques génériques de communication dans la technique et les supports, la communication territoriale présente néanmoins d'autres enjeux : l'intérêt général et la démocratie. Les valeurs publiques, dont la transparence, s'y déclinent (Jorgensen, Bozeman, 2002) : cette communication «tend à l'échange et au partage d'informations d'utilité publique ainsi qu'au maintien du lien social» (Zemor, 2008 : 5). Elle a un double objectif, que la technologie Internet sert parfaitement : rendre compte des politiques menées (les contraintes et les arbitrages) et rechercher l'adhésion des administrés.

Les années 1990 voient la naissance de la « COM FI», l'arrivée d'Internet et la reprise des émissions obligataires. Confrontées à des exigences financières comme le sont les sociétés cotées, les collectivités émettrices présentent-elles une utilisation comparable des outils numériques pour leur communication financière ? Cette communication financière numérique diffère-t-elle de celle des collectivités non émettrices ? Est-il possible ou souhaitable de construire une communication financière numérique propre aux collectivités émettrices?

Conformément à la littérature, nous montrerons pourquoi les collectivités émettrices devraient mener une communication financière numérique semblable à celle des sociétés cotées (1). Nous dresserons un état des lieux des pratiques des collectivités, sur le contenu des sites Internet et sur l'exploitation de la technologie numérique (2). Des préconisations sont alors avancées pour tendre vers un véritable management de la relation avec les investisseurs (3). 


\section{Le financement des collectivités territoriales par les marchés va influencer leur communication financière sur Internet}

La communication financière des collectivités locales (1.1) ne peut pas rester inchangée face à une reprise des financements directs (1.2), les marchés exigeant des émetteurs une diffusion d'information sur Internet (1.3). La littérature permet l'élaboration d'un modèle et d'hypothèses de recherche (1.4).

\subsection{La communication territoriale : institutionnelle et financière}

La loi ATR de 1992 crée une impulsion. Si les collectivités ont plutôt orienté leurs sites Internet vers des préoccupations institutionnelles et politiques, elles ont aussi cerné les enjeux d'une communication financière (Huron, Serdeczny, 2002) : recherche de transparence et exercice de démocratie, légitimation des décisions publiques, évaluation des actions menées. Par ailleurs, les «transformations progressives de la comptabilité publique en France (M14, LOLF...) répondent à des besoins forts d'évolution de l'information financière des organisations publiques au service du management des organisations publiques » (Lande, Rocher, 2008). À côté des contrôles de légalité et de régularité exercés par les services de l'État, le contrôle d'opportunité se heurte à la libre administration des collectivités territoriales ${ }^{2}$ et positionne l'exécutif local dans une véritable communication. Celle-ci devient un outil de maximisation de l'utilité de l'élu de la théorie du Public Choice. C'est aussi un accès facilité au financement externe : une information continue crée la confiance (Angotti, Laurent, 2000).

\subsection{Vers un financement accru des collectivités sur les marchés}

La difficulté d'emprunter est une réalité pour toute collectivité locale dans la France de 2011. Les banques resserrent l'accès au crédit : moins de réponses aux appels, offres sur un montant partiel, marges croissantes, durées d'endettement réduites. Les établissements bancaires sont eux-mêmes dans la tourmente : exigence de solvabilité accrue, respect des normes de Bâle 3, moindre rentabilité suite à l'arrêt de la commercialisation de produits structurés. La tendance est telle que les exécutifs territoriaux envisagent une diversification de leurs sources de financement, via le projet de création d'une agence de financement des investissements locaux (AFIL) et l'émission d'obligations (Pays de la Loire, Limousin, Auvergne, Rhône-Alpes entre 2009 et 2011).

Il s'agit d'un financement prisé par les collectivités dès le XIX ${ }^{\mathrm{e}}$ siècle (Berthon, GallaisHamonno, 1994), redevenu d'actualité avec la notation financière et le rehaussement de crédit dans les années 1990. Ces émissions obligataires sont complétées depuis $2001^{3}$ par des Titres de Créances Négociables : Billets de Trésorerie et Bons à Moyen Terme Négociables ${ }^{4}$. Les émissions d'obligations font encore figure d'exception à côté du crédit bancaire (3\%

\footnotetext{
${ }^{2}$ Art. 72 de la Constitution.

${ }^{3}$ Loi sur les Nouvelles Régulations Économiques du 15 mai 2001.

${ }^{4}$ D'un jour à un an pour les BT; au-delà d'un an pour les BMTN.
} 
de l'encours total ${ }^{5}$ ), mais tout porte à croire qu'il s'agit du début d'une tendance de fond, tournée vers le financement direct. La question de la communication financière de ces nouveaux émetteurs est posée.

\subsection{L'exigence d'information des marchés}

La théorie financière met en lumière l'asymétrie d'information entre les dirigeants de la firme et ses actionnaires (Leland, Pyle, 1977). Les responsables de l'entreprise, en charge de bons projets d'investissement, doivent impérativement se signaler aux marchés de capitaux, pour leur permettre d'anticiper une espérance de performance attractive et valoriser les titres de la société (Paturel et al., 2006). Selon la théorie du signal, la structure financière de la firme (dont l'endettement) constitue un signal envoyé aux marchés (Ross, 1977). Avide d'informations, le marché exige alors une politique de communication efficace. Elle consiste d'abord à porter à la connaissance de l'investisseur, l'existence même de l'émetteur, puis de lui permettre une évaluation continue de son risque.

«La pratique de la communication financière consiste pour une société cotée en bourse, à assurer la promotion auprès des acteurs des marchés financiers des valeurs mobilières qu'elle émet : actions, obligations ou produits hybrides. Elle doit les aider à ce qu'ils puissent ainsi établir leur juste valeur." (Guimard, 2007 : 5). Les objectifs des collectivités émettrices sont similaires (Léger, 2008) : répondre aux obligations légales, dans une perspective de protection de l'épargnant. «Il s'agit pour la collectivité de renseigner les investisseurs extérieurs sur la nature du projet à financer en réduisant ainsi l'asymétrie d'information » (Gourmel-Rouger, 1998 : 206). En ce sens, l'émission de titres constitue en elle-même, un signal et génère une réputation. Si la levée de capitaux sur les marchés exige une communication, elle est aussi à elle seule une communication. Au-delà du gain financier, il s'agit de «la recherche ou l'assise d'une notoriété ou d'une reconnaissance » (Gourmel-Rouger, 2000 : 53). La communication financière sert l'émission et l'émission sert la communication institutionnelle.

Le cadre de la théorie du signal (Evans, Patton, 1987) et de l'agence peut être transposé au secteur public. Une relation d'agence fait alors de l'électeur le principal, et de l'élu l'agent maximisant un gain qui dépend de sa réélection et du respect de promesses électorales (Laswad et al., 2005). Les exécutifs ont intérêt à une diffusion volontaire de l'information, contrôlant et valorisant leur action. Ce constat est d'autant plus prégnant que la compétition politique ou électorale s'intensifie. Le parallèle doit néanmoins rester incomplet car les collectivités territoriales sont uniquement émettrices de titres de créance. La question de l'endettement reste posée (Ingram, 1984) : les élus construisent une communication financière pour abaisser le coût de la dette et dégager des marges de manœuvre.

Pour partager toute l'information instantanément et servir un objectif de réputation dans une finance mondialisée, le média Internet apparaît incontournable. En ouvrant le champ des possibles, Internet aura révolutionné la communication financière des sociétés cotées (Léger, 2008). La littérature relève l'impact de divers déterminants comme, dans un cadre international, la taille, la cotation des titres, la technologie (Debreceny et al., 2002),

\footnotetext{
5 Selon le rapport d'enquête parlementaire sur les produits financiers à risque souscrits par les acteurs publics locaux de décembre 2011.
} 
l'environnement (Xiao, 2004), ou encore la dispersion de la structure de propriété et le statut juridique ( $\mathrm{Li}, 2010)$ dans le cadre national.

Des outils similaires sont désormais mis en œuvre à l'échelon public local (Laswad et al., 2005). L'information est disponible en temps réel et devient universelle. La technologie élargit la nature des supports (écrit, audio, vidéo) et permet surtout l'interactivité. Comparativement à un support papier, son faible coût matériel est enfin un avantage indéniable ${ }^{6}$. Internet a été introduit au même moment dans les entreprises et les collectivités locales mais il existe aujourd'hui une grande diversité dans les contenus et usages, particulièrement en matière de communication financière.

\subsection{Modèle de recherche et hypothèses : une plus forte diffusion d'informations financières sur le Net pour les collectivités émettrices}

Du fait d'un recours accru au marché et conformément à l'exigence d'information qui en découle, nous analysons la communication financière des collectivités locales sur Internet en testant l'hypothèse selon laquelle les collectivités recourant à des émissions de titres (dites «émettrices ») renforcent leur diffusion d'informations financières sur Internet par rapport à celles qui n'émettent pas (dites « non émettrices »).

Cette hypothèse générique est déclinée en contenus financiers, en exploitation de l'outil Internet et en une présentation des émissions, en vertu d'une littérature existante sur la communication financière volontaire des entreprises (tableau 1).

En H1, nous supposons que les collectivités émettrices, à l'instar des sociétés cotées, rendent disponibles davantage de contenus. D'une part (E1), les états financiers devraient être présentés : les comptes annuels et l'information financière essentielle (Almilia, Budisusetyo, 2008) (1 à 4), mais aussi des outils plus élaborés, comme des ratios financiers et des rapports d'audit ou d'analyse (Dutta, Bose, 2007) (5 à 7). D'autre part (E2), la transposition aux finances locales est impérative : la procédure budgétaire devrait rester centrale dans la communication des collectivités. En outre, les budgets sont tout à la fois le lien avec la politique menée par l'exécutif (cf. stratégie globale dans l'entreprise, Léger, 2008) et des résultats intermédiaires (Dutta, Bose, 2007) (8 à 10). Nous pouvons imaginer quelques prolongements : la mise en ligne du Débat d'Orientation Budgétaire (cf. assemblée générale, Léger, 2008) (11), une page Web dédiée et un téléchargement possible (Pervan, 2006) (12 et 13), nouer une relation avec l'administré-investisseur (cf. avec l'actionnaire, Dutta, Rose, 2007) (14).

En H2, nous faisons l'hypothèse que les sites des émettrices devraient exploiter davantage les potentialités du numérique (Arnone et al., 2010). D'une part (E3), une page Web spécifique devrait apparaitre dans les sites institutionnels (Pervan, 2006) (15 et 16), un contact spécifique par courriel notamment (Barredy, Darras, 2008) (17 et 18) auprès du fonctionnaire et de l'élu en charge des affaires financières. D'autre part (E4), les sites des émettrices devraient davantage exploiter les potentialités numériques (Arnone et al.2010) (19 à 21), des liens vers des évaluations externes (Dutta, Rose, 2007) (22) et vers un site dédié (Léger, 2008) (23), l'animation de forums (Barredy, Darras, 2008) (24 et 25), en intégrant la nature itérative et interactive du support (Leclerc, Ruette-Guyot, 2009).

\footnotetext{
${ }^{6}$ En faisant abstraction de coûts indirects et du risque de réputation, en cas de mauvaise utilisation de l'outil numérique.
} 
En H3, nous nous attendons à trouver sur le site des émettrices des informations relatives aux obligations et TCN émis (E5), dont les prospectus AMF (Léger, 2008) (26), mais aussi une page Web dédiée (Pervan, 2006) (27), un site à destination des investisseurs (Leger, 2008) (28) et un lien vers un site de cotation (cf. historiques de cours et dividendes pour des actions, Barredy, Darras, 2008) (29).

Tableau 1 : Hypothèses et variables du modèle

\begin{tabular}{|c|c|}
\hline & H1 - Contenus de communication financière supérieurs chez les émettrices \\
\hline \multirow[b]{2}{*}{ 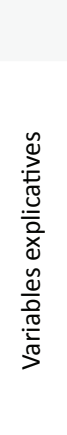 } & Variable expliquée - E1- Présence des états financiers Optique "Documents" \\
\hline & $\begin{array}{l}1 \text { Le rapport annuel ou rapport d'activité } 2010 \text { est disponible } \\
2 \text { Le rapport financier ou rapport CA } 2010 \text { est disponible } \\
3 \text { Le compte administratif } 2010 \text { est disponible } \\
4 \text { L'état de la dette contenu dans l'annexe au CA } 2010 \text { est disponible } \\
5 \text { Les ratios ATR } 2010 \text { sont explicitement présentés } \\
6 \text { Il est fait état d'un rapport d'audit (cabinet, Chambre Régionale des Comptes...) } \\
7 \text { II est fait état d'un rating }\end{array}$ \\
\hline \multirow{3}{*}{ 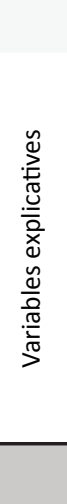 } & Variable expliquée - E2- Présence des évolutions budgétaires Optique "Risques" \\
\hline & $\begin{array}{l}8 \text { Le Budget Primitif } 2011 \text { est disponible } \\
9 \text { Les décisions modificatives ou le Budget Supplémentaire } 2010 \text { sont disponibles } \\
10 \text { Au moins un budget annexe } 2011 \text { est disponible } \\
11 \text { Le Débat d’Orientation Budgétaire } 2011 \text { est relaté } \\
12 \text { II existe un commentaire du budget } 2011 \text { sur une page dédiée } \\
13 \text { Une plaquette "budget } 2011 \text { " est téléchargeable } \\
14 \text { Présence d'un numéro du magazine édité par la collectivité consacré au budget } 2011\end{array}$ \\
\hline & H2- Contenu Internet du site supérieur chez les émettrices \\
\hline \multirow[b]{2}{*}{ 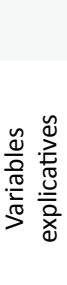 } & Variable expliquée - E3- Ergonomie du site Optique "visite du site" \\
\hline & $\begin{array}{l}15 \text { Il existe un onglet budget/finance } \\
16 \text { Taper "dette"/“emprunt" dans le moteur de recherche mène à une information pertinente } \\
17 \text { Contact direct avec responsable/le service des finances par mail } \\
18 \text { Contact direct avec l'élu en charge des finances/la commission des finances par mail }\end{array}$ \\
\hline
\end{tabular}




\begin{tabular}{|c|c|}
\hline & Variable expliquée - E4- Exploitation des potentialités du Web Optique "valeur ajoutée d'Internet" \\
\hline \multirow[t]{2}{*}{ 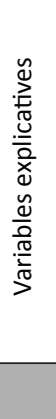 } & $\begin{array}{l}19 \text { II existe des documents financiers d'un autre format que le format écrit : audio, vidéo } \\
20 \text { Actualisations du site (flux RSS) } \\
21 \text { Une traduction (en anglais) des informations financières est disponible } \\
22 \text { II existe des liens vers des évaluations/données externes (Chambre Régionale des Comptes, } \\
\text { Bercy, DGCL) } \\
23 \text { II existe un lien vers un site dédié au budget } \\
24 \text { II existe une tribune des groupes politiques ou un forum de discussion (on peut y parler finances) } \\
25 \text { II existe un accès à des réseaux sociaux (Facebook, Twitter) }\end{array}$ \\
\hline & H3 - Présence d'Information sur les émissions \\
\hline \multirow[b]{2}{*}{ 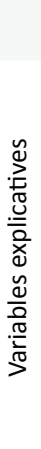 } & Variable expliquée E5 - ÉMISSION DE TITRES - Optique “infos sur les émissions” \\
\hline & $\begin{array}{l}26 \text { Le document présentant l'émission est disponible } \\
\text { à savoir : le prospectus de l'émission obligataire } \\
\text { à savoir : le dossier de présentation financière de l'émission de TCN } \\
27 \text { Taper "émission obligataire" ou "émission TCN" dans le moteur de recherche mène à une } \\
\text { information pertinente } \\
28 \text { II existe un lien vers un site dédié aux investisseurs } \\
29 \text { Il existe un lien vers un site de cotation des titres } \\
\text { à savoir : le site d'Euronext ou un autre marché de cotation (pour les émissions obligataires) } \\
\text { ou l'AMF } \\
\text { à savoir : le site de la Banque de France (pour les émissions de TCN) }\end{array}$ \\
\hline
\end{tabular}

\section{2. État des lieux de la communication financière des collectivités territoriales sur Internet}

Pour tester le modèle de recherche, une double analyse (quantitative et qualitative) est menée sur notre population de collectivités émettrices, ainsi que sur une population de non émettrices, construite par pairage (2.1). Les résultats des analyses quantitative (2.2) et qualitative (2.3) sont présentés et interprétés au regard de la littérature (2.4).

\subsection{Cadre méthodologique : du pairage aux analyses quantitative et qualitative}

L'ambition étant de mettre en évidence l'utilisation faite de l'Internet en cas de recours aux marchés de capitaux, il faut pouvoir comparer les pratiques des collectivités émettrices et celles des non émettrices. Pour ce faire, il faut disposer de deux populations, distantes d'un seul fait distinctif : l'émission.

D'une part, les émetteurs d'obligations sont identifiés via la base de données Fininfo ${ }^{7}$ et les émetteurs de billets de trésorerie, à partir des dossiers de présentation financière

\footnotetext{
${ }^{7}$ Extraction de la base de Six Telekurs au 07/06/2011, à laquelle a été ajoutée l'émission obligataire de la Région Limousin du 13 juin 2011.
} 
de la Banque de France ${ }^{8}$ pour établir une liste exhaustive des collectivités émettrices à l'été 2011.

D'autre part, puisque l'évaluation des sites des émettrices nécessite la constitution d'un échantillon témoin de collectivités non émettrices, via une technique de pairage (révélant différences et ressemblances, cf. études sur des sociétés - Pozniak, Croquet, 2011). Les non émettrices doivent présenter des caractéristiques similaires, susceptibles d'orienter la communication financière : besoins de financement, moyens disponibles, réalité fiscale et réalité de la dette (éléments figurant dans les onze ratios $\mathrm{ATR}^{9}$, tableau 2).

Tableau 2 : Les ratios ATR comme critères de pairage

\begin{tabular}{|c|c|l|l|}
\hline Facteur & Ratio* & \multicolumn{1}{|c|}{ Définition } & \multicolumn{1}{c|}{ Interprétation } \\
\hline Besoins & $n^{\circ} 10$ & $\begin{array}{l}\text { = dépenses d'équipement brut/ } \\
\text { recettes réelles de fonctionnement }\end{array}$ & Effort d'équipement/richesse de la collectivité \\
\hline Moyens & $n^{\circ} 9$ & $\begin{array}{l}\text { fonctionnement + remboursement } \\
\text { de dette)/recettes réelles de } \\
\text { fonctionnement }\end{array}$ & $\begin{array}{l}\text { Marge d'autofinancement courant : capacité à } \\
\text { financer l'investissement après paiement des } \\
\text { charges obligatoires }\end{array}$ \\
\hline Fiscalité & $\begin{array}{c}\mathrm{n}^{\circ} 8 / 8 \\
\text { bis* }\end{array}$ & $=$ recettes fiscales/potentiel fiscal & $\begin{array}{l}\text { Coefficient de mobilisation du potentiel } \\
\text { fiscal : rapport entre le produit fiscal effectif } \\
\text { et théorique, mesure du niveau de pression } \\
\text { fiscale }\end{array}$ \\
\hline Dette & $\mathrm{n}^{\circ} 11$ & $\begin{array}{l}\text { Comparaison de la charge de la dette à la } \\
\text { richesse de la collectivité }\end{array}$ \\
\hline
\end{tabular}

* Source : DGCL. Ratios de 2007 pour les communes; ratios de 2009 pour les départements et régions.

** pour les communes : fiscalité directe encaissée au titre de la commune et du groupement à fiscalité propre.

Pour chaque paire, il faut minimiser la distance émettrice - non émettrice (de même strate) sur ces quatre ratios, en excluant un écart trop grand sur l'un d'entre eux. La pertinence des paires obtenues (tableau 3 ) est ensuite validée par des tests des signes et de Wilcoxon.

\footnotetext{
${ }^{8}$ www.banque-france.fr au 20/07/2011. Seuls des billets de trésorerie sont émis par les collectivités françaises (pas dossier de présentation financière de BMTN à cette date).

${ }^{9}$ Loi du 6 février 1992 sur l'Administration Territoriale de la République. Données obligatoires pour les régions, départements et communes de plus de 10000 habitants.
} 
Tableau 3 : Les émettrices et leurs paires

\begin{tabular}{|c|c|c|c|}
\hline & Émettrices & $\begin{array}{c}\text { Non Émettrices } \\
\text { Associées }\end{array}$ & Nature des émissions \\
\hline Régions & $\begin{array}{l}\text { Champagne-Ardenne } \\
\text { lle-de-France* } \\
\text { Limousin } \\
\text { Nord-Pas-de-Calais } \\
\text { Pays-de-la-Loire } \\
\text { Provence-Alpes-Côte-d'Azur } \\
\text { Rhône-Alpes }\end{array}$ & $\begin{array}{l}\text { Franche-Comté } \\
\text {---- } \\
\text { Centre } \\
\text { Auvergne } \\
\text { Lorraine } \\
\text { Bourgogne } \\
\text { Picardie }\end{array}$ & $\begin{array}{l}\text { Obligations } \\
\text { Obligations + BT** } \\
\text { Obligations } \\
\text { Obligations + BT } \\
\text { Obligations + BT } \\
\text { BT } \\
\text { BT }\end{array}$ \\
\hline Départements & $\begin{array}{l}\text { Essonne } \\
\text { Seine-et-Marne }\end{array}$ & $\begin{array}{l}\text { Var } \\
\text { Alpes-Maritimes }\end{array}$ & $\begin{array}{l}\text { Obligations + BT } \\
\text { Obligations + BT }\end{array}$ \\
\hline Communes & $\begin{array}{l}\text { Aubagne } \\
\text { Bordeaux } \\
\text { Boulogne-Billancourt } \\
\text { Lyon } \\
\text { Marseille } \\
\text { Paris* } \\
\text { Tours }\end{array}$ & $\begin{array}{l}\text { Villiers-sur-Marne } \\
\text { Rennes } \\
\text { Aix-en-Provence } \\
\text { Clermont-Ferrand } \\
\text { Saint-Étienne } \\
\text {---- } \\
\text { Perpignan }\end{array}$ & $\begin{array}{l}\text { Obligations } \\
\text { Obligations } \\
\text { BT } \\
\text { Obligations + BT } \\
\text { Obligations } \\
\text { Obligations + BT } \\
\text { Obligations }\end{array}$ \\
\hline
\end{tabular}

* Aucun pairage possible du fait de la singularité de ces collectivités

** Billets de Trésorerie

Le cadre méthodologique réside en premier lieu en une analyse quantitative, à savoir un scoring des sites, pour juger de la distance (dans les contenus et l'utilisation faite du numérique) entre émettrices et non émettrices. Ceci est prolongé en second lieu, par une approche complémentaire (puisque qualitative) pour donner du sens aux constats dressés, dépasser les agrégations parfois trop influencées par les plus faibles scores et raisonner sur une différenciation des contenus. L'analyse qualitative permet en outre, de faire émerger les éléments nécessaires à la formulation de préconisations plus fines, à destination du praticien.

\subsection{Approche quantitative : création d'un scoring des sites}

\section{Méthodologie : comparaison des scores}

Conformément aux hypothèses, la méthodologie repose sur l'évaluation des sites des collectivités par la création d'un scoring. Dans la grille d'analyse, la présence de chaque variable explicative est affectée d'un point, leur somme attribuant un score ${ }^{10}$. Des tests non paramétriques vérifient ensuite s'il existe une différence significative entre les scores obtenus par les émettrices et les non émettrices sur chaque item.

\section{Résultats : contenus financiers légèrement plus riches pour les émettrices, sans une meilleure exploitation des potentialités des sites Internet}

Globalement, tant sur les contenus que sur l'utilisation du numérique, les scores ne sont pas très différents, notamment dans les documents budgétaires (E2) et les potentialités techniques des sites (E4). Ce premier constat appelle une lecture plus fine.

\footnotetext{
${ }^{10}$ Sur la période du 26 septembre au 17 octobre 2011.
} 
Conformément au tableau 4, les seuls écarts positifs significatifs sont relevés sur les états financiers : une présentation du rating et du dernier compte administratif est plus fréquente sur le site des émettrices.

Tableau 4 : Comparaison du scoring $\mathrm{H} 1$

\begin{tabular}{|c|c|c|c|c|c|c|c|c|}
\hline \multicolumn{9}{|c|}{ Synthèse scoring H1 - CONTENUS DE COMMUNICATION FINANCIÈRE } \\
\hline $\begin{array}{l}\text { Score émettrices } \\
\text { > score non } \\
\text { émettrices }\end{array}$ & W & Sig & $\begin{array}{c}\text { Score émettrices = } \\
\text { score non émettrices }\end{array}$ & $\mathbf{W}$ & Sig & $\begin{array}{c}\text { Score émettrices } \\
\text { < score non } \\
\text { émettrices }\end{array}$ & W & Sig \\
\hline $\begin{array}{l}\text { Variable expliquée } \\
\text { E1 } \\
\text { ÉTATS FINANCIERS } \\
\text { - Optique } \\
\text { "Documents" }\end{array}$ & & & $\begin{array}{l}\text { Rapport annuel ou } \\
\text { rapport d'activité } 2010 \\
\text { disponible }\end{array}$ & NS & NS & & & \\
\hline $\begin{array}{l}\text { CA } 2010 \text { disponible } \\
\text { Il est fait état d'un } \\
\text { rating }\end{array}$ & $\mathrm{S}$ & $\begin{array}{l}\text { NS } \\
\text { S }\end{array}$ & $\begin{array}{l}\text { Rapport financier } \\
\text { ou rapport CA } 2010 \\
\text { disponible } \\
\text { État de la dette } 2010 \\
\text { disponible } \\
\text { Ratios ATR } 2010 \\
\text { explicitement présentés } \\
\text { Il est fait état d'un } \\
\text { rapport d'audit }\end{array}$ & $\begin{array}{l}\text { NS } \\
\text { NS } \\
\text { NS } \\
\text { NS }\end{array}$ & $\begin{array}{l}\text { NS } \\
\text { NS } \\
\text { NS } \\
\text { NS }\end{array}$ & & & \\
\hline $\begin{array}{l}\text { Variable expliquée } \\
\text { E2 } \\
\text { ÉVOLUTIONS } \\
\text { BUDGÉTAIRES } \\
\text { - Optique } \\
\text { "Risques" } \\
\text { Plaquette } \\
\text { "budget 2011" } \\
\text { téléchargeable }\end{array}$ & NS & NS & $\begin{array}{l}\text { BP } 2011 \text { disponible } \\
\text { Décisions modificatives } \\
\text { Budget Supplémentaire } \\
2010 \text { disponibles } \\
\text { Au moins un budget } \\
\text { annexe } 2011 \text { disponible } \\
\text { Commentaire du budget } \\
2011 \text { sur une page } \\
\text { dédiée } \\
\text { Numéro du magazine } \\
\text { consacré au budget } 2011\end{array}$ & $\begin{array}{l}\text { NS } \\
\text { NS } \\
\text { NS } \\
\text { NS } \\
\text { NS }\end{array}$ & $\begin{array}{l}\text { NS } \\
\text { NS } \\
\text { NS } \\
\text { NS } \\
\text { NS }\end{array}$ & DOB 2011 relaté & NS & NS \\
\hline
\end{tabular}

W : test de Wilcoxon; Sig : Test des Signes; S/NS : Significatif/Non Significatif au seuil de $5 \%$ 
D'après le tableau 5, les écarts sont plutôt négatifs sur le fonctionnement du site et l'exploitation des potentialités des sites. Mais au final, seul le moteur de recherche pour les mots « dette/emprunt » est plus pertinent.

Tableau 5 : Comparaison du scoring $\mathrm{H} 2$

\begin{tabular}{|c|c|c|c|c|c|c|c|c|}
\hline \multicolumn{9}{|c|}{ Synthèse scoring H2- CONTENUS INTERNET } \\
\hline $\begin{array}{l}\text { Score émettrices > } \\
\text { score non émettrices }\end{array}$ & w & Sig & $\begin{array}{c}\text { Score } \\
\text { émettrices = } \\
\text { score non } \\
\text { émettrices }\end{array}$ & w & Sig & $\begin{array}{l}\text { Score émettrices < } \\
\text { score non émettrices }\end{array}$ & w & Sig \\
\hline $\begin{array}{l}\text { Onglet budget/finance } \\
\text { Contact direct de l'élu } \\
\text { en charge des finances } \\
\text { par mail }\end{array}$ & 更 & 更 & & & & $\begin{array}{l}\text { Variable expliquée E3 } \\
\text { ERGONOMIE DU SITE - } \\
\text { Optique "visite du site" } \\
\text { Moteur de recherche } \\
\text { pertinent pour « dette/ } \\
\text { emprunt " } \\
\text { Contact direct de l'élu } \\
\text { en charge des finances } \\
\text { par mail }\end{array}$ & $\begin{array}{l}\text { S } \\
\text { NS }\end{array}$ & $\begin{array}{l}\text { S } \\
\text { NS }\end{array}$ \\
\hline & & & $\begin{array}{l}\text { Flux RSS } \\
\text { Liens vers } \\
\text { évaluations/ } \\
\text { données } \\
\text { externes } \\
\\
\text { Réseaux } \\
\text { sociaux }\end{array}$ & 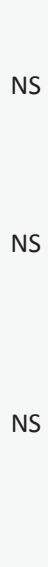 & NS & $\begin{array}{l}\text { Variable expliquée E4 } \\
\text { EXPLOITATION DES } \\
\text { POTIENTIALITÉS DU } \\
\text { NET - Optique "valeur } \\
\text { ajoutée d'Internet" } \\
\text { Documents audio-vidéo } \\
\text { Traduction en anglais } \\
\text { Lien vers site dédié au } \\
\text { budget } \\
\text { Tribune des groupes } \\
\text { politiques ou un forum } \\
\text { de discussion }\end{array}$ & $\begin{array}{l}\text { NS } \\
\text { NS } \\
\text { NS } \\
\text { NS }\end{array}$ & $\begin{array}{l}\text { NS } \\
\text { NS } \\
\text { NS } \\
\text { NS }\end{array}$ \\
\hline
\end{tabular}

W : test de Wilcoxon; Sig : Test des Signes; S/NS : Significatif/Non Significatif au seuil de $5 \%$

Les résultats du scoring donnent un avantage aux émettrices sur les contenus financiers, mais pas sur l'usage des potentialités numériques, s'éloignant des pratiques des sociétés cotées. Ce premier constat est complété par une étude qualitative des contenus, de surcroît parce que le score des émettrices est sévèrement influencé par les quelques sites les moins performants.

\subsection{Approche qualitative : analyse textuelle des sites}

\section{Méthodologie : principe de l'analyse textuelle}

Conduite avec le logiciel d'analyse textuelle Alceste (Analyse des Lexèmes Cooccurents dans un Ensemble de Segments de Texte), la méthode découpe le corpus à analyser en une suite de segments de texte pour y observer la distribution des mots (Reiner, 2007). L'analyse 
textuelle automatisée repose sur une « déstructuration » du corpus (Gavard-Perret, HelmeGuizon, 2008) pour retenir la répétition des mots (les cooccurrences).

Le corpus traité par Alceste est segmenté en Unités de Contexte Élémentaire (notées UCE). Cette méthode ne classifie pas des mots, mais des UCE et procède à une classification descendante hiérarchique. Alceste calcule alors la décomposition du corpus en différentes classes et parmi toutes les décompositions possibles, maximise les oppositions (Khi2 des marges). À l'issue du traitement, le corpus est ainsi « rangé » en différentes classes.

Les éléments à analyser doivent impérativement être des documents écrits relatifs aux finances et au budget, communs aux émettrices et non émettrices et disponibles sur les sites Internet en lecture directe ou en téléchargement. Le choix s'est ainsi porté sur les éléments suivants : page dédiée au budget 2011, plaquette sur le budget 2011, rapport financier 2010 ou rapport sur CA 2010, partie « finances » du rapport annuel 2010 et tout contenu sur le rating.

Des Unités de Contexte Initiales ou UCI (variables étoiles) sont définies par l'utilisateur qui indique une partition du corpus en fonction des individus et de leurs caractéristiques ce qui favorise un pré-découpage naturel du corpus et permet un croisement ultérieur des modalités de variables caractérisant les individus avec le corpus. Ainsi, pour analyser l'usage fait des fonctionnalités d'Internet, les variables utilisées dans le scoring ont été définies comme variables étoiles.

Résultats : contenus financiers nettement plus riches pour les émettrices et avance toute relative dans l'exploitation des fonctionnalités d'Internet

Les mots et les variables étoiles (dont la présence ou l'absence est significative) repérés dans chaque classe conduisent respectivement à la définition de thématiques et à l'identification d'utilisations du Net (tableau 6).

Tableau 6 : Analyse des variables étoiles « émettrices » vs « non émettrices »

\begin{tabular}{|c|c|c|c|c|c|c|}
\hline \multirow{2}{*}{$\begin{array}{l}\text { Intitulé des } \\
\text { classes }\end{array}$} & \multicolumn{2}{|c|}{$\begin{array}{l}\text { Variables propres } \\
\text { aux émettrices }\end{array}$} & \multicolumn{2}{|c|}{ Variables communes } & \multicolumn{2}{|c|}{$\begin{array}{l}\text { Variables propres aux } \\
\text { non émettrices }\end{array}$} \\
\hline & $\begin{array}{l}\text { Présence } \\
\text { significative }\end{array}$ & $\begin{array}{c}\text { Absence } \\
\text { significative }\end{array}$ & $\begin{array}{c}\text { Présence } \\
\text { significative }\end{array}$ & $\begin{array}{c}\text { Absence } \\
\text { significative }\end{array}$ & $\begin{array}{l}\text { Présence } \\
\text { significative }\end{array}$ & $\begin{array}{c}\text { Absence } \\
\text { significative }\end{array}$ \\
\hline $\begin{array}{c}\text { Gestion } \\
\text { de la dette }\end{array}$ & $\begin{array}{l}\text { Onglet budget } \\
\text { performant } \\
\text { Flux RSS } \\
\text { Forum }\end{array}$ & $\begin{array}{l}\text { Adresse du } \\
\text { Responsable } \\
\text { de Service }\end{array}$ & $\begin{array}{l}\text { Moteur de } \\
\text { recherche } \\
\text { performant }\end{array}$ & & $\begin{array}{l}\text { Réseaux } \\
\text { sociaux }\end{array}$ & $\begin{array}{l}\text { Adresse } \\
\text { de l'élu } \\
\text { Forum }\end{array}$ \\
\hline Fiscalité & $\begin{array}{l}\text { Onglet budget } \\
\text { performant }\end{array}$ & $\begin{array}{l}\text { Adresse du } \\
\text { Responsable } \\
\text { de Service }\end{array}$ & & $\begin{array}{l}\text { Adresse } \\
\text { de l'élu }\end{array}$ & $\begin{array}{l}\text { Adresse } \\
\text { responsable } \\
\text { service } \\
\text { Réseaux } \\
\text { sociaux }\end{array}$ & $\begin{array}{l}\text { Onglet } \\
\text { budget } \\
\text { Audio-vidéo }\end{array}$ \\
\hline
\end{tabular}




\begin{tabular}{|c|c|c|c|c|c|}
\hline $\begin{array}{c}\text { Gestion } \\
\text { budgétaire }\end{array}$ & $\begin{array}{l}\text { Onglet budget } \\
\text { performant } \\
\text { Moteur de } \\
\text { recherche } \\
\text { performant } \\
\text { Audio-vidéo }\end{array}$ & $\begin{array}{l}\text { Adresse } \\
\text { de l'élu } \\
\text { Adresse du } \\
\text { Responsable } \\
\text { de Service } \\
\text { Flux RSS }\end{array}$ & & & \\
\hline $\begin{array}{c}\text { Actions } \\
\text { économiques }\end{array}$ & $\begin{array}{l}\text { Flux RSS } \\
\text { Présence d'un } \\
\text { forum } \\
\text { Réseaux } \\
\text { sociaux }\end{array}$ & Audio-vidéo & $\begin{array}{l}\text { Onglet } \\
\text { budget } \\
\text { performant } \\
\text { Moteur de } \\
\text { recherche } \\
\text { performant } \\
\text { Adresse } \\
\text { de l'Elu }\end{array}$ & Audio-vidéo & \\
\hline $\begin{array}{l}\text { Services aux } \\
\text { administrés }\end{array}$ & $\begin{array}{l}\text { Moteur de } \\
\text { recherche } \\
\text { non } \\
\text { performant } \\
\text { Adresse } \\
\text { responsable } \\
\text { service }\end{array}$ & $\begin{array}{l}\text { Audio-vidéo } \\
\text { Flux RSS } \\
\text { Forum }\end{array}$ & & & \\
\hline $\begin{array}{c}\text { Projets } \\
\text { d'urbanisme }\end{array}$ & $\begin{array}{l}\text { Adresse } \\
\text { responsable } \\
\text { service } \\
\text { Réseaux } \\
\text { Sociaux }\end{array}$ & & & & \\
\hline $\begin{array}{c}\text { Actions } \\
\text { culturelles }\end{array}$ & $\begin{array}{l}\text { Moteur de } \\
\text { recherche } \\
\text { non } \\
\text { performant } \\
\text { Adresse } \\
\text { responsable } \\
\text { service }\end{array}$ & $\begin{array}{l}\text { Audio-vidéo } \\
\text { Forum } \\
\text { Réseaux } \\
\text { sociaux }\end{array}$ & & & \\
\hline $\begin{array}{l}\text { Administration } \\
\text { de l'institution }\end{array}$ & & & & $\begin{array}{l}\text { Adresse } \\
\text { de l'élu } \\
\text { Forum }\end{array}$ & $\begin{array}{l}\text { Réseaux } \\
\text { sociaux }\end{array}$ \\
\hline
\end{tabular}

Propre aux émettrices, la classe « gestion budgétaire » valide l'hypothèse H1 d'un contenu financier plus grand. Sur l'usage des potentialités numériques (H2), les émettrices semblent en avance dans les classes « gestion de la dette » et «fiscalité », avec un onglet dédié au budget et un forum de discussion. Le cadre d'hypothèses faisant des collectivités des émetteurs semblables aux sociétés cotées semble néanmoins devoir être dépassé. En effet, d'autres thématiques non financières émergent des discours : il s'agit d'une communication institutionnelle, visant la construction d'un sentiment identitaire pour les citoyens et la promotion du territoire pour les acteurs économiques. La classe « services aux administrés » est typiquement une «communication de proximité » (Megard, Deljarrie, 2009). 


\subsection{Discussion des résultats : quels retours sur la littérature antérieure?}

Les résultats révèlent une proximité avec les pratiques des sociétés émettrices : présenter leurs comptes (Almilia, Budisusetyo, 2008), tenir un discours sur la procédure budgétaire (Léger, 2008), diffuser leur rating (Dutta, Bose, 2007) et faire de leur note une communication (Gourmel-Rouger, 2000). L'hypothèse H1, selon laquelle le contenu de la communication financière des émettrices est supérieure, est validée au regard de ces variables explicatives.

Les émettrices ne semblent pas faire un meilleur usage de la technologie, contrairement à ce qui était supposé (H2) pour les pratiques des sociétés (Arnone et al., 2010). Par exemple, nous ne trouvons pas systématiquement de communication sur la dette dans les communications institutionnelles (Pervan, 2006). Néanmoins, la présence significative d'un forum de discussion accrédite l'idée selon laquelle l'émission conduit à une réflexion sur la dette et des débats avec l'opposition ou les administrés, en conformité avec la nécessité des échanges (Barredy, Darras, 2008) et l'interactivité (Leclerc, Ruette-Guyot, 2009). Ces éléments incitent à ne pas rejeter l'hypothèse H2 selon laquelle les émettrices utilisent plus largement la technologie numérique, mais plutôt à suggérer une validation partielle. En outre, la communication budgétaire, ancêtre d'une communication financière « moderne » (Klopfer, 2010), propose des documents audio-vidéo, comme si, conformément à la chronologie des pratiques (Laurent, 1994), la richesse des supports débutait par la « communication budgétaire » pour atteindre à terme la « communication financière ».

Contrairement aux entreprises, les collectivités valorisent davantage la destination de leurs dépenses que les perspectives de recettes. Les émettrices communiquent sur d'autres thèmes que les seules problématiques financières : actions économiques, services offerts aux administrés, projets d'urbanisme, actions culturelles ${ }^{11}$. Elles sont des collectivités locales avant d'être des émettrices. Elles véhiculent des valeurs publiques, dont la transparence (Bozeman, 2007). L'élu local n'est pas que le clientéliste ou l'utilitariste court-termiste décrit par l'école du Public Choice : il «porte des valeurs, est dépositaire d'une identité, a une culture politique » (Sine, 2005 : 23). Enfin, la garantie apportée par l'État français, donc un risque estimé peu élevé (en général rating AA), n'incite pas jusqu'à présent à une communication financière très développée.

Il s'agit vraisemblablement du début d'un processus, que la nécessité de trouver des financements dans un contexte de crise financière va accélérer et que nous proposons d'orienter par la formulation de préconisations.

\section{Préconisation pour une contribution à un « Bondholder Relationship Management ॥}

Si une communication sur Internet s'impose pour toute émission de titre de créance (3.1), des préconisations plus fines sont formulées au regard de la loi ATR (3.2) et des événements obligataires (3.3). Enfin, le clivage dans l'utilisation des potentialités numériques ne s'explique pas en totalité par le statut émettrice/non émettrice.

\footnotetext{
${ }^{11}$ Les non émettrices se distinguent par une classe sur l'administration de l'institution, non commentée ici car éloignée des préoccupations de communication financière.
} 


\subsection{Emprunt obligataire et communication financière numérique}

Les collectivités locales accèdent aux marchés de capitaux après l'obtention du visa de l'Autorité des Marchés Financiers (AMF) sur une notice d'information (prospectus), via un placement public ${ }^{12}$ ou un placement privé : il s'opère soit par les administrés du territoire émetteur, devenus financeurs, soit par un contrat de souscription entre l'émetteur et un investisseur institutionnel ${ }^{13}$.

Les préconisations concernent ici le placement auprès des habitants. Des arguments de démocratie et de transparence rencontrent l'optique d'une épargne locale voire citoyenne. L'administré était contribuable et usager, il devient investisseur. Cibler l'investisseur individuel répond aussi à un calcul financier : plus fidèle, moins exigeant sur la rentabilité, que l'investisseur institutionnel (dit actif et supportant plus de coûts de transaction) (Coutton, 2008). Pour toutes ces raisons, développer le placement auprès du grand public devrait se révéler un pari intéressant.

Internet paraît le chemin le plus court vers le citoyen souscripteur. Ainsi, la démarche est celle d'un visiteur du site, intéressé par l'investissement dans des titres de créance émis par la collectivité (pas celle du Webmaster - Guimard, 2007). À l'heure du cybermarketing financier et du SRM pour Shareholder Relationship Management dans les entreprises (Léger, 2008), l'objectif est de contribuer à la réflexion sur un Bondholder Relationship Management, qui moderniserait la gestion de la relation entre investisseurs et collectivités émettrices.

Une exploitation plus pertinente des fonctionnalités d'Internet est recherchée pour les émettrices, en rapportant leurs scores aux notes moyennes (5 points sur les 10 attribués par rubrique de E1 à E5) et en analysant les contenus propres aux émissions.

\subsection{Les insuffisances révélées par le scoring : valoriser les obligations ATR et s'inspirer des pratiques sociétaires}

La première recommandation (tableau 7) faite aux collectivités territoriales est l'utilisation des potentialités d'Internet pour répondre aux obligations légales en matière d'information financière. La loi ATR introduit la publication de ratios financiers, instaure le Débat d'Orientation Budgétaire et consacre la notion de « groupe collectivité locale », élargissant la communication financière aux « satellites ». Sur ces trois seuls éléments, l'étude révèle des scores inférieurs à la moyenne des points attribués. Or, il s'agit de points fondamentaux pour l'investisseur. Une simple «transposition Web» de ces obligations est ainsi proposée : ratios de la collectivité présentés et comparés aux ratios de la strate, sources de liens externes vers les sites ministériels de l'Intérieur ou des Finances ${ }^{14}$. Le Débat d'Orientation Budgétaire pourrait prendre une dimension vidéo, expliquant le budget voté, mais exposant aussi la force avec laquelle il est défendu par les élus. Les investisseurs seraient enfin sensibles à de l'information sur tous les budgets annexes, dans l'optique d'une véritable évaluation du risque de solvabilité de l'émetteur, et à terme, à une communication financière consolidée. À cette fin, Internet offrirait la possibilité de télécharger les différents documents, dans un archivage numérique.

\footnotetext{
${ }^{12}$ Qui s'opère directement ou via un syndicat de banques par une prise ferme.

${ }^{13}$ Les emprunts sont le plus souvent effectués auprès d'investisseurs institutionnels.

${ }^{14}$ www.colloc.bercy.gouv.fr ou www.dgcl.interieur.gouv.fr
} 
Tableau 7 : Synthèse score H1 par rapport au score moyen

\begin{tabular}{|c|c|c|}
\hline \multicolumn{3}{|c|}{ Synthèse scoring $\mathrm{H} 1$ - contenus de communication financière } \\
\hline $\begin{array}{l}\quad \text { Variables pour } \\
\quad \text { lesquelles le } \\
\text { score émettrices > } \\
\text { score moyen }\end{array}$ & $\begin{array}{l}\text { Variables pour } \\
\text { lesquelles le } \\
\text { score émettrices } \\
\approx \text { score moyen }\end{array}$ & $\begin{array}{l}\text { Variables pour lesquelles } \\
\text { le score émettrices < score moyen }\end{array}$ \\
\hline $\begin{array}{l}\text { Il est fait état d'un } \\
\text { rating }\end{array}$ & 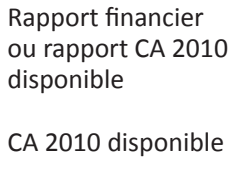 & $\begin{array}{l}\text { E1 - ÉTATS FINANCIERS - Optique «Documents» } \\
\text { Rapport annuel ou rapport d'activité } 2010 \text { disponible } \\
\text { État de la dette } 2010 \text { disponible } \\
\text { Ratios ATR } 2010 \text { explicitement présentés } \\
\text { Il est fait état d'un rapport d'audit }\end{array}$ \\
\hline $\begin{array}{l}\text { Commentaire } \\
\text { budget } 2011 \text { sur } \\
\text { une page dédiée }\end{array}$ & $\begin{array}{l}\text { BP } 2011 \text { disponible } \\
\text { Plaquette } \\
\text { «budget 2011» } \\
\text { téléchargeable }\end{array}$ & $\begin{array}{l}\text { E2 - ÉVOLUTIONS BUDGÉTAIRES - Optique «Risques» } \\
\text { Décisions modificatives ou Budget Supplémentaire } 2010 \\
\text { disponibles } \\
\text { Au moins un budget annexe } 2011 \text { disponible } \\
\text { DOB } 2011 \text { relaté } \\
N^{\circ} \text { du magazine consacré au budget } 2011\end{array}$ \\
\hline
\end{tabular}

Les pratiques des sociétés cotées pourraient être transposées aux émetteurs publics locaux : accessibilité systématique d'un rapport annuel, état de la dette, liens vers des évaluations externes du risque de crédit (rapports d'analyse des agences de notation, rapports d'audit de cabinets indépendants, de la Chambre Régionale des Comptes), possibilité de joindre directement par émail le responsable du service des finances (tableau 8) pour faire du courrier électronique, « un point nodal structurant dans le portefeuille technologique » (Tran, $2010: 200$ ) des financiers territoriaux.

Tableau 8 : Synthèse score H2 par rapport au score moyen- contenus Internet

\begin{tabular}{|c|c|c|}
\hline \multicolumn{3}{|c|}{ Synthèse scoring $\mathrm{H} 2$ - contenus Internet } \\
\hline $\begin{array}{l}\text { Variables pour lesquelles } \\
\text { le score émettrices > score } \\
\text { moyen }\end{array}$ & $\begin{array}{l}\text { Variables pour lesquelles le score } \\
\text { émettrices } \approx \text { score moyen }\end{array}$ & $\begin{array}{c}\text { Variables pour lesquelles } \\
\text { le score émettrices < score } \\
\text { moyen }\end{array}$ \\
\hline $\begin{array}{l}\text { Onglet budget/finance } \\
\text { Moteur de recherche pertinent } \\
\text { pour « dette/emprunt " }\end{array}$ & $\begin{array}{l}\text { E3- ERGONOMIE DU SITE - Optique } \\
\text { "visite du site» } \\
\text { Contact direct de l'élu en charge des } \\
\text { finances par mail }\end{array}$ & $\begin{array}{l}\text { Contact direct du responsable } \\
\text { du service des finances par } \\
\text { mail }\end{array}$ \\
\hline $\begin{array}{l}\text { Flux RSS } \\
\text { Réseaux sociaux }\end{array}$ & $\begin{array}{l}\text { Documents audio-vidéo } \\
\text { Traduction en anglais } \\
\text { Liens vers des évaluations/données } \\
\text { externes } \\
\text { Lien vers site dédié au budget } \\
\text { Tribune des groupes politiques ou forum } \\
\text { de discussion }\end{array}$ & $\begin{array}{l}\text { E4 - Optique EXPLOITATION } \\
\text { DES POTIENTIALITÉS DU } \\
\text { NET - } \\
\text { "valeur ajoutée d'Internet" }\end{array}$ \\
\hline
\end{tabular}


Enfin, pour l'émission stricto sensu (tableau 9), il faut reproduire les pratiques des sociétés cotées : mise à disposition du prospectus AMF, lien vers un site de cotation et idéalement, un site dédié aux investisseurs. La région Bourgogne montre la voie avec un site exclusivement consacré au budget, accessible depuis son site institutionnel.

Tableau 9 : Synthèse score H3 par rapport au score moyen

\begin{tabular}{|c|l|l|}
\hline \multicolumn{2}{|c|}{ Synthèse scoring H3- contenus spécifique émission } \\
\hline $\begin{array}{c}\text { Variables pour } \\
\text { lesquelles le } \\
\text { score émission } \\
>\text { score moyen }\end{array}$ & $\begin{array}{c}\text { Variables pour lesquelles le } \\
\text { score émission } \approx \text { score } \\
\text { moyen }\end{array}$ & \multicolumn{1}{c|}{$\begin{array}{c}\text { Variables pour lesquelles le } \\
\text { score émission < score moyen }\end{array}$} \\
\hline & $\begin{array}{c}\text { Moteur de recherche } \\
\text { pertinent pour « émission } \\
\text { obligataire ou TCN" }\end{array}$ & $\begin{array}{l}\text { E5 - ÉMISSION DE TITRES - Optique «infos sur } \\
\text { les émissions» } \\
\text { Lien vers site dédié aux investisseurs } \\
\text { Document présentant l'émission disponible } \\
\text { Lien vers site de cotation des titres }\end{array}$ \\
\hline
\end{tabular}

\subsection{L'évaluation du contenu textuel des émissions : promouvoir l'opération et aller vers le Web 2.0}

Une seconde analyse textuelle est réalisée sur les documents relatifs à l'émission (disponibles sur les sites ou en téléchargement) : prospectus, page dédiée, communiqué de presse. Seul un tiers des collectivités émettrices est étudié ${ }^{15}$, les autres ne proposant aucun des éléments cités.

Les classes (tableau 10) et leurs intitulés tracent le cadre imposé par un prospectus d'émission : les collectivités locales sont soumises aux mêmes règles que tous les emprunteurs obligataires ${ }^{16}$, il y a bien adaptation du message à la cible.

\footnotetext{
${ }^{15}$ Essonne, Limousin, Nord Pas-de-Calais, Paris, Pays de la Loire.

${ }^{16}$ Règles régissant l'émission de titres, l'appel public à l'épargne, et l'admission à la cote : loi du 3 janvier 1983 sur le développement des investissements et la protection de l'épargne (cf. art. L1611-3 du Code Général des Collectivités Territoriales).
} 
Tableau 10 : Analyse des variables étoiles « émissions »

\begin{tabular}{|c|c|}
\hline Intitulé des classes & Variables propres aux émissions \\
\hline Projet à financer & Moteur de recherche performant \\
\hline $\begin{array}{l}\text { L'émetteur du point de vue } \\
\text { Financier }\end{array}$ & $\begin{array}{l}\text { Adresse élu } \\
\text { Pas d'audio-vidéo } \\
\text { Pas de forum } \\
\text { Réseaux sociaux }\end{array}$ \\
\hline Optique Investisseur & $\begin{array}{l}\text { Pas d'adresse élu } \\
\text { Présence d'audio-vidéo } \\
\text { Présence d'un forum }\end{array}$ \\
\hline $\begin{array}{l}\text { Aspects réglementaires de } \\
\text { l'émission }\end{array}$ & $\begin{array}{l}\text { Pas d'adresse élu } \\
\text { Présence d'audio-vidéo } \\
\text { Présence d'un forum } \\
\text { Pas de réseaux sociaux }\end{array}$ \\
\hline Montage de l'émission & $\begin{array}{l}\text { Pas d'adresse élu } \\
\text { Présence d'audio-vidéo } \\
\text { Présence d'un forum } \\
\text { Pas de réseaux sociaux }\end{array}$ \\
\hline
\end{tabular}

Les variables confortent les résultats du scoring et conduisent à s'interroger sur une utilisation encore trop timide des potentialités d'Internet : peu de réseaux sociaux, pas de liens externes, pas de site en anglais, pas d'information pour contacter le service des finances, autant d'éléments manquants pour parler de réelle communication financière sur Internet.

L'interactivité permise par le Web 2.0 devrait être systématisée, « via des outils tels que les espaces de concertation numérique (chats et forums) [pour] récolter plus rapidement de l'information, comme les réactions à des projets » (Huron, Spieth, 2009 : 116), pour être mise au service d'une épargne locale, d'investissements financés pour et par les habitants du territoire. Les émissions obligataires riment avec endettement attractif, prise d'indépendance face aux banques et communication institutionnelle. Celle-ci n'apparaît pas ici ${ }^{17}$ (ni forum, ni dimension audio-vidéo). Nous préconisons donc la valorisation de l'émission sur le site. Si «Marseille est un exemple de l'utilisation de ce mode de financement à des fins de communication » (Serve, 2002 : 139), cet exercice n'est pas continu sur le Web : prônons le « ritualisme » de cette «position de communication » (Rivière, 2010).

\subsection{Vers un autre clivage dans I'utilisation des potentialités d'Internet pour la communication financière des collectivités territoriales}

L'analyse textuelle conduite en fusionnant les corpus des émettrices et celui des non émettrices fait ressortir six classes. Quatre d'entre elles (« urbanisme », « culture », « action économique », «fiscalité ») présentent sensiblement les mêmes caractéristiques qu'au sein des analyses menées séparément sur les deux groupes. L'intérêt du résultat réside dans l'existence de deux classes (tableau 11) : «budget » et « dette », classiques pour la communication financière publique locale (Michel-Clupot, Rouot, 2012).

\footnotetext{
${ }^{17}$ À l'instar de l'émission de la région Rhône-Alpes, dont l'émission d'octobre 2011 n'est pas mentionnée sur son site.
} 
Tableau 11 : Analyse des variables étoiles « émettrices » ET « non émettrices »

\begin{tabular}{|c|l|l|}
\hline & \multicolumn{1}{|c|}{ Budget } & \multicolumn{1}{|c|}{ Dette } \\
\hline \multirow{4}{*}{$\begin{array}{c}\text { Collectivités } \\
\text { territoriales }\end{array}$} & $\begin{array}{l}\text { Tours } \\
\text { Paris } \\
\text { Aix-en-Provence } \\
\text { Marseille }\end{array}$ & $\begin{array}{l}\text { Essonne } \\
\text { Champagne-Ardenne } \\
\text { Rhône-Alpes } \\
\text { Limousin } \\
\text { Boulogne-Billancourt } \\
\text { Picardie }\end{array}$ \\
\hline \multirow{5}{*}{$\begin{array}{l}\text { Variables } \\
\text { Internet }\end{array}$} & $\begin{array}{l}\text { Onglet budget/finance } \\
\text { Moteur de recherche pertinent } \\
\text { pour « dette/emprunt » } \\
\text { Pas de contact direct avec le responsable } \\
\text { du service des finances } \\
\text { Pas de contact direct avec l'élu } \\
\text { Documents audio-vidéo } \\
\text { Pas de flux RSS } \\
\text { Pas de forum }\end{array}$ & $\begin{array}{l}\text { Moteur de recherche pertinent } \\
\text { pour « dette/emprunt » } \\
\text { Pas de contact direct avec le responsable } \\
\text { du service des finances } \\
\text { Pas de contact direct avec l'élu } \\
\text { Présence d'un flux RSS } \\
\text { Présence d'un forum } \\
\text { Réseaux sociaux }\end{array}$ \\
\hline
\end{tabular}

Des villes et une communication budgétaire s'opposent à des régions et une communication financière. En outre, les premières sont dans un usage élémentaire des fonctionnalités d'Internet, simple support de diffusion d'une information existant par ailleurs (E3 du scoring). Les secondes s'inscrivent dans une exploitation plus complète de l'interactivité du Web 2.0 (E4 du scoring). Si l'exercice de la démocratie locale doit se faire sur l'arbitrage impôt/emprunt comme sur les choix techniques d'une gestion active de l'encours (Michel, 2001), il en est de même pour l'information de l'investisseur.

Une communication traditionnelle sur le budget local conduite par des maires s'oppose à une forme plus moderne de communication sur la gestion de la dette menée par les régions (échelon territorial beaucoup plus récent, en charge du développement économique et en prise directe avec les milieux économiques). Les deux classes regroupent indifféremment des émettrices et des non émettrices.

Ainsi, le clivage «émettrices/non émettrices »n'explique pas les divergences dans l'utilisation des potentialités numériques, en matière de communication financière. Issues des travaux sur l'audit dans le secteur public, des pistes peuvent être esquissées : l'intensité de la compétition politique (Baber, 1983), la temporalité dans un mandat (Carassus, Laborde, 2002). L'introduction d'une dimension politique ne pourrait cependant se faire que dans une diversité de couleurs politiques à la tête des territoires et sur la totalité d'un cycle électoral.

Si le clivage « présentation budgétaire/stratégie financière » s'avère plus pertinent, cette distinction entre tradition et modernité dans les contenus se retrouve également dans l'utilisation faite des Technologies de l'Information et de la Communication. Dans les entreprises, "l'information comptable et légale a cédé très largement la place à une communication financière active, volontaire maîtrisée et ciblée » (Léger, 2008). Si les collectivités territoriales semblent suivre le même chemin sur les contenus de communication financière, nous sommes en droit d'imaginer une tendance similaire dans l'usage des fonctionnalités d'Internet, vers plus d'interactivité entre émetteurs et investisseurs. 


\section{Conclusion}

Cet article montre que les pratiques de communication financière sur Internet des collectivités émettrices sont encore bien éloignées de celles des entreprises cotées en Bourse. Le scoring des sites révèle un peu plus de contenus financiers pour les émettrices, mais pas un recours important aux innovations numériques, résultat conforté par l'analyse qualitative.

Les collectivités émettrices se comportent avant tout comme des collectivités. Le facteur discriminant est moins l'émission que le degré de maturité de la communication : budgétaire ou financière. La présente approche est explicitement financière mais pourrait être complétée par une approche plus politique ou porteuse de valeurs publiques, afin d'interpréter les pratiques de communication financière sur Internet. Ces perspectives supposent néanmoins d'autres méthodologies ou a minima, un contexte politique présentant plus de diversité.

Trente ans après la décentralisation, 20 ans après la loi ATR et 10 ans après les sites institutionnels, des préconisations sont formulées pour de nouveaux usages des potentialités d'Internet, répondant aux exigences d'un financement désintermédié : donner une dimension numérique à la loi ATR, aller vers les pratiques des sociétés cotées, faire entrer l'investisseur dans l'interactivité du Web 2.0.

Les collectivités locales sont au début d'un long processus : face à la difficulté d'emprunter, elles vont devoir diversifier leurs sources de financement au profit d'émissions de titres de créance. Le projet de création d'une agence de financement de l'investissement local est un premier signal fort à destination des marchés. La communication doit désormais façonner l'image financière de la collectivité territoriale dans le cadre d'une stratégie intégrant un Bondholder Relationship Management.

\section{Bibliographie}

ALMILIA L., BUDISUSETYO S., (2008). The Practice of Financial Disclosure on Corporate Website : Case Study in Indonesia, Proceeding International Conference on Business \& Mangement, University Brunel Darussalem, 8-9 january 2008.

ANGOTTI P., LAURENT P., (2000). La communication financière des collectivités locales : enjeux et perspectives, Revue Française de Finances Publiques 71, 9-24.

ARNONE L., COLOT O., CROQUET M., GEERTS A., POZNIAK L., (2010). Internet comme vecteur de communication financière : une analyse des entreprises du marché libre, La Revue des Sciences de Gestion, Direction et Gestion 242, 49-55.

BABER W. R., (1983). Toward Understanding the Role of Auditing in the Public Sector, Journal of Accounting and Economics 5, 213-227.

BARREDY C., DARRAS N., (2008). L'utilisation d'Internet dans la communication auprès des actionnaires minoritaires dans les entreprises familiales cotées, Journal des entreprises familiales 1 (1), 41-68.

BERTHON J., GALLAIS-HAMONNO G., (1994). Les collectivités territoriales peuvent-elles être des acteurs financiers de plein exercice sur les marchés monétaires et obligataires ? Revue d'Economie Financière, Actes du séminaire du 2 décembre 1994, 89-103.

BOZEMAN B., (2007). La publicitude normative : comment concilier valeurs publiques et valeurs du marché, Politiques et management public 25 (4), 179-211. 
CARASSUS D., LABORDE D., (2002). L'impact politique de l'audit de début de mandat, in Technologie et management de l'information : enjeux et impacts dans la comptabilité, le contrôle et l'audit, Actes du colloque de l'AFC, mai 2002.

COUTTON D., (2008). Quelle stratégie développer pour une communication financière réussie? Décisions Marketing 50, 71-73.

CURIEN N., MAXWELL W., (2011). La neutralité d'Internet, La Découverte, Paris.

DEBRECENY R., GRAY G., RAHMAN A., (2002). The Determinants of Internet Financial Reporting, Journal of Accounting and Public Policy 21, 371-394.

DUTTA P., BOSE S., (2007). Web-Based Corporate Reporting in Bangladesh : An Exploratory Study, the Cost and Management 35 (6), 29-45.

EVANS J., PATTON J., (1987). Signaling and Monitoring in Public-Sector Accounting, Journal of Accounting Research 25 Supplement 1987.

GAVARD-PERRET M-L., HELME-GUIZON A., (2008). Choisir parmi les techniques spécifiques d'analyse qualitative, In Méthodologie de la recherche, Pearson Education, Paris, 247-279.

GOURMEL-ROUGER C.,(1998). Les déterminants du choix d'endettement des grandes collectivités territoriales françaises : une étude empirique sur la période 1985-1995, Revue d'économie financière 47, 187-212.

GOURMEL-ROUGER C., (2000). Émissions obligataires des collectivités territoriales françaises : le choix de la notation ou du rehaussement de crédit, Banque et Marchés 47, 44-53.

GUIMARD A., (2007). La communication financière. Théorie et pratique, Economica Paris.

HURON D., SPIETH G., (2009). Communication intercommunale : entre construction d'image du territoire et modification du comportement des usagers, Communication \& Organisation 35, 112-122.

HURON D., SERDECZNY V., (2002). La communication budgétaire et financière des communes sur Internet : obligations ou opportunités? XVIe Journées nationales des IAE Paris, septembre 2002.

INGRAM R., (1984) Economic Incentives and the Choice of State Government Accounting Practices, Journal of Accounting Research 22 (1), 126-144.

JORGENSEN T., BOZEMAN B., (2002). Public Values Lost? Public Management Review.4 (1), 63-81.

KLOPFER M., (2010). Gestion financière des collectivités locales, $5^{\mathrm{e}}$ Ed, Le Moniteur, Paris.

LANDE E., ROCHER S., (2008). 25 ans de réforme comptable au service du management public, Politiques et management public 26 (3), 25 ans de politiques et de management public.

LASWAD F., FISHER R., OYELERE P., (2005). Determinants of Voluntary Internet Financial Reporting by Local Government Authorities, Journal of Accounting and Public Policy 24, 101-121.

LAURENT P., (1994). La communication financière des collectivités locales, condition et conséquence de la désintermédiation, Revue d'Economie Financière Actes du séminaire du 2 décembre 1994, 105-111.

LECLERC S., RUETTE-GUYOT E, (2009).Web 2.0 : la communication iter-@ctive Economica, Paris.

LÉGER J-Y., (2008). La communication financière, $2^{\mathrm{e}}$ Ed, Dunod, Paris.

LELAND H., PYLE D., (1977). Information Asymetries, Financial Structures and Financial Intermediaries, Journal of Finance 32, 371-387.

LI L., (2010). L'offre d'information volontaire par Internet et le gouvernement des entreprises françaises, Finance Contrôle Stratégie 13 (4), 101-137.

MEGARD D., DELJARRIE B., (2009). La communication des collectivités locales, $2^{\mathrm{e}}$ Ed, LGDJ, Paris.

MICHEL M., (2001). Quelle démocratie pour la gestion de la dette des collectivités locales? In Démocratie et management local Dalloz, Paris, 373-385.

MICHEL-CLUPOT M., ROUOT S., (2012). Communication financière des collectivités locales françaises et crise internationale : des pratiques en mutation, Politiques et Management Public 29 (3), 343-367.

PATUREL R., MATOUSSI H., JOUINI S., (2006). Les motivations de la communication financière des entreprises françaises et britanniques à travers le Web, Colloque international de l'AFFI, Poitiers, juin. 
PERVAN I., (2006). Voluntary Financial Reporting on the Internet - Analysis of the Practice of Croatian and Slovene Listed Joint Stock Companies, Financial Theory and Practice 30 (1), 1-27.

POZNIAK L., CROQUET M., (2011). Les déterminants de la communication financière sur Internet : le cas des marchés non réglementés de Bruxelles, Journal of Small Business and Entrepreneurship 24 (3), 329-344.

REINERT M., (2007). Analyse statistique de données textuelles en sciences de gestion Editions EMS, Collombelles.

ROSS S., (1977). The Determination of Capital Structure: the Incentive Signalling Approach, the Bell Journal of Economics 40, 23-40.

RIVIERE A., (2010). Politique de réduction des coûts et communication financière : ritualisme, opportunisme ou nomadisme? Comptabilité Contrôle Audit tome XVI, vol 2, 127-158.

SERVE S. (2002)., Le recours au financement désintermédié par une collectivité locale et l'évaluation de la prime de risque obligataire : le cas de la ville de Marseille, Finance Contrôle Stratégie 5 (2), 107-142.

TRAN S., (2010). Quand les TIC réussissent trop bien dans les organisations : le cas du courrier électronique chez les managers, Revue Management \& Avenir 34 (4), 200-215.

XIAO J., YANG H., CHOW C., (2004). The Determinants and Characteristics of Voluntary Internet-Based Disclosures by Listed Chinese Companies, Journal of Accounting and Public Policy 23, 191-225.

ZEMOR P., (2008). La communication publique, 4 e édition, Que sais-je? PUF, Paris.

\section{Annexe : Webographie utilisée}

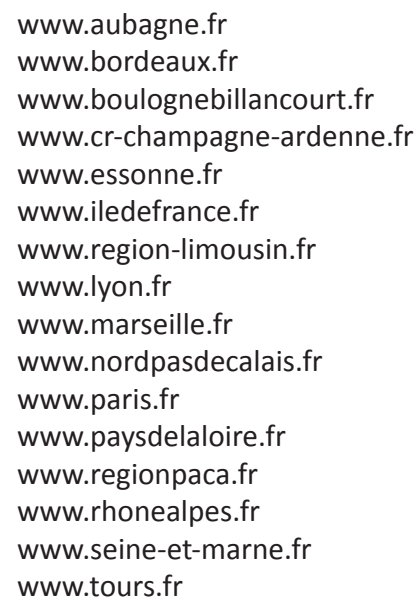

www.cg06.fr

www.mairie-aixenprovence.fr

www.auvergne.org

www.region-bourgogne.fr

www.regioncentre.fr

www.clermont-ferrand.fr

www.franche-comte.fr

www.lorraine.eu

www.mairie-perpignan.fr

www.picardie.fr

www.rennes.fr

www.saint-etienne.fr

www.var.fr

www.mairie-villiers94.com 